\title{
Photoionization yield of atomic hydrogen using intense few-cycle pulses
}

\author{
O. Ghafur ${ }^{1,2}$, W. C. Wallace ${ }^{1,2}$, J. E. Calvert ${ }^{1,2}$, D. E. Laban $^{1,2}$, M. G. Pullen ${ }^{1,2}$, A. N. Grum- \\ Grzhimailo $^{3,4}$, K. Bartschat ${ }^{4}$, I. V. Litvinyuk ${ }^{2}$, R. T. Sang ${ }^{1,2}$, and D. Kielpinski ${ }^{1,2} *$ \\ ${ }^{1}$ ARC Centre of Excellence for Coherent X-Ray Science, Griffith University, Nathan, QLD, 4111, Australia \\ ${ }^{2}$ Australian Attosecond Science Facility and Centre for Quantum Dynamics, Griffith University, Nathan, QLD, 4111 \\ Australia \\ ${ }^{3}$ Department of Physics and Astronomy, Drake University, Des Moines, Iowa, USA \\ ${ }^{4}$ Institute of Nuclear Physics, Moscow State University, Moscow, Russia
}

Synopsis We present experimentally measured photoionization yields of atomic hydrogen as a function of laser intensity for few-cycle laser pulses. Comparison of data with exact ab-initio simulations produce better agreement than analytical theories and enable accurate intensity calibration.

The interaction of intense few-cycle infrared laser pulses with matter is the fundamental process at the heart of attosecond and strong-field science [1]. The complex, highly nonlinear dynamics that occur in the regime of few-cycle laser pulses necessitate accurate theoretical simulations in order to retrieve useful physical measurements and provide a sensible physical interpretation of the experimental data.

Strong-field ionization experiments involving atomic hydrogen $(\mathrm{H})$ have been previously performed [2,3], however agreement with theory was predominately qualitative. Building on our earlier work, which obtained quantitative agreement at the $10 \%$ level between simulations and measurements of photoelectron spectra in $\mathrm{H}$ [4], we now extend this scheme to measurements of the total photoionization yield.

The experimental apparatus consists of a fewcycle laser pulse interacting with an $\mathrm{H}$ beam. Our commercial "Femtolaser Compact Pro" laser produces linearly polarized, $6.0 \mathrm{fs}, 800 \mathrm{~nm}$ central wavelength, $\sim 150 \mu \mathrm{J}$ light pulses at a repetition rate of $1 \mathrm{kHz}$. The $\mathrm{H}$ beam is created by collisional dissociation of $\mathrm{H}_{2}$ in a radiofrequency discharge powered by a helical resonator. An off-axis parabolic mirror is used to focus the laser beam into the $\mathrm{H}$ beam, near the entrance to a $\sim 10 \mathrm{~cm}$ field-free time-of-flight mass spectrometer. We measure the $\mathrm{H}$ ion yield as a function of laser intensity for two different focal spot sizes of $\sim 45 \mu \mathrm{m}$ and $\sim 75 \mu \mathrm{m}$.

Our data (Figure 1) have been fit to results from direct integration of the non-relativistic time-dependent Schrödinger equation (TDSE), and to results from the commonly used analytical tunneling theory of Ammosov-DeloneKrainov (ADK) [6]. The figure illustrates a failure of the simpler model in describing the accu-

\footnotetext{
*E-mail: d.kielpinski@gmail.com
}

rate ionization for even the simplest atomic system. Fitting the $\mathrm{H}$ yield with ab-initio theory is a simple method of accurately obtaining the intensity dependent yield for any other species present in the experiment.

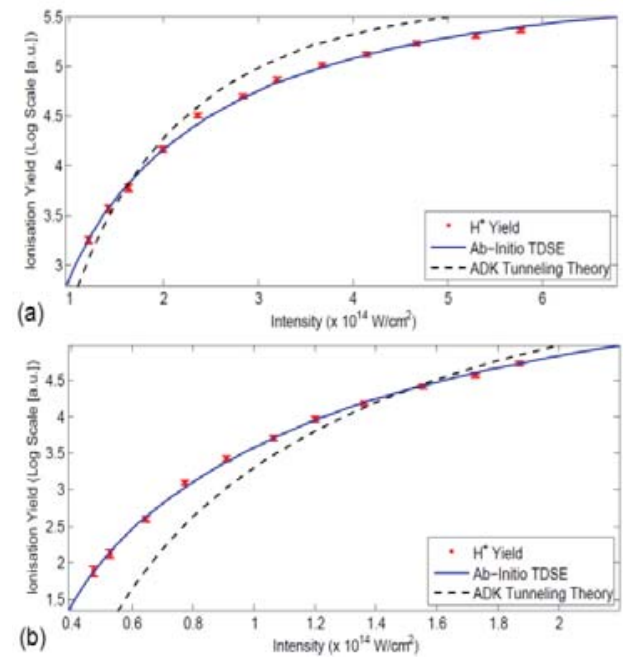

Figure 1. Measured $\mathrm{H}$ yield (points) as a function of laser intensity with 6 fs pulses with a focal spot of $\sim 45 \mu \mathrm{m}$ (a), and $\sim 75 \mu \mathrm{m}$ (b). Fits to the TDSE simulations (solid line) [5] has been used to calibrate the intensity scale. Corresponding predictions for the ADK simulations (dashed lines) are plotted alongside for comparison.

\section{References}

[1] P. B. Corkum \& F. Krausz 2007 Nat. Phys. 6381

[2] H. Rottke et al 1990 Phys. Rev. Lett. 64404

[3] G. G. Paulus et al 1996 J. Phys. B: At. Mol. Opt. Phys. 29 L249

[4] M. G. Pullen et al 2011 Opt. Lett. 363660

[5] A. N. Grum-Grzhimailo et al 2010 Phys.Rev. A. 81043408

[6] M. V. Ammosov et al 1965 Sov. Phys. JETP 64 1307 